\title{
Enhancing $\mathrm{U}_{\text {eff }}$ in Oxime-Bridged [ $\mathrm{Mn}_{6}^{\mathrm{III}} \mathrm{Ln}^{\mathrm{III}}{ }_{2}$ ] Hexagonal Prisms
}

\author{
Guillame Rigaux, ${ }^{a, b}$ Ross Inglis, ${ }^{b}$ Susan Morrison, ${ }^{b}$ Alessandro Prescimone, ${ }^{b}$ Cyril Cadiou, ${ }^{b}$ Marco \\ Evangelisti*c $^{c}$ and Euan K. Brechin* ${ }^{b}$
}

\author{
Received (in $X X X, X X X)$ Xth $X X X X X X X X X 200 X$, Accepted Xth $X X X X X X X X X 200 X$ \\ ${ }_{5}$ First published on the web Xth $X X X X X X X X X 200 X$ \\ DOI: $10.1039 / b 000000 x$
}

The first 3d-4f clusters built using derivatised salicylaldoximes $\left(\mathrm{R}\right.$-saoH $\left.{ }_{2}\right)$ describe unusual hexagonal prisms. Replacement of the paramagnetic Gd(III) ions with diamagnetic $\operatorname{Ln}($ III) ions 10 allows for a more thorough understanding of the magnetic properties, whilst replacement with $\mathrm{Tb}(\mathrm{III})$ doubles $\mathbf{U}_{\text {eff- }}$

The re-ignition of interest in the synthesis of polymetallic $3 d$ 4f clusters $^{1}$ originates from two major sources: singlemolecule magnetism ${ }^{2}$ and molecular cooling. ${ }^{3}$ One way to 15 increase the effective energy barrier to magnetisation reversal in the former class of compounds is through the introduction of highly anisotropic f-block ions such as Dy(III), Tb(III), Ho(III) or $\operatorname{Er}(\mathrm{III})^{4}$ since it has been shown that even single-ion complexes containing these metals can display hysteresis 20 loops in magnetisation versus field studies. ${ }^{5}$ High spin isotropic molecules with weak exchange interactions, on the other hand, are ideal candidates for enhanced magnetic cooling $^{6}$ since the negligible anisotropy permits easy polarisation of the net molecular spin leading to large changes 25 in magnetic entropy, and the presence of degenerate or lowlying excited spin states results in extra magnetic entropy from the so-added degrees of freedom. The Gd(III) ion is thus an ideal choice for building molecular refrigerants. ${ }^{7}$

Since derivatised salicylaldoxime ligands $\left(\mathrm{R}-\mathrm{saoH}_{2}\right.$; Figure 30 1) have been remarkably successful in the mediation of ferromagnetic exchange interactions between $\mathrm{Mn}$ (III) ions and thus in the synthesis of high spin $\mathrm{Mn}(\mathrm{III})$ molecules, $^{8}$ a sensible extension to this synthetic strategy is the incorporation of lanthanide ions into the clusters. ${ }^{9}$ While the 35 coordinative flexibility of $\mathrm{Ln}(\mathrm{III})$ ions makes the prediction of molecular structure even more difficult, it is likely that any high-spin Mn(III)-Ln(III) cluster built with these ligands will be an SMM or magnetic cooler. Herein we report the first examples of $3 d-4 f$ complexes employing R-sao ${ }^{2-}(\mathrm{R}=\mathrm{Me}, \mathrm{Et})$ 40 - unusual [ $\mathrm{Ln}_{2}^{\mathrm{III}}{ }_{2} \mathrm{Mn}_{6}^{\mathrm{III}}{ }_{6}$ ] $(\mathrm{Ln}=\mathrm{Gd}, \mathrm{La}, \mathrm{Tb})$ hexagonal prisms.

Reaction of $\mathrm{Gd}(\mathrm{acac})_{3} \cdot 2 \mathrm{H}_{2} \mathrm{O}, \mathrm{Mn}\left(\mathrm{NO}_{3}\right)_{2} \cdot 6 \mathrm{H}_{2} \mathrm{O}$, Et-saoH and $\mathrm{NaOH}$ in a 1:1 mixture of $\mathrm{MeCN} / \mathrm{MeOH}$ for 2 hours, followed by filtration and slow evaporation of the mother liquor affords black crystals of $\left[\mathrm{Gd}_{2} \mathrm{Mn}_{6} \mathrm{O}_{3}(\mathrm{OMe})_{4}(\mathrm{Et}-\right.$ $\left.{ }_{45} \mathrm{SaO}_{6}(\mathrm{acac})_{2}(\mathrm{MeOH})_{4}\right] \cdot 0.7 \mathrm{MeCN}(\mathbf{1} \cdot 0.7 \mathrm{MeCN}$; Figure 1) in the monoclinic space group $\mathrm{P} 2{ }_{1} / \mathrm{c}^{\dagger \ddagger}$ The metallic core contains a non-planar (chair-like) wheel of six $\mathrm{Mn}(\mathrm{III})$ ions capped top and bottom by a Gd(III) ion, such that the skeleton describes a distorted hexagonal prism (Figure 1). The Gd(III) 50 ions are connected to the $\mathrm{Mn}$ (III) ions via three $\mu_{4}-\mathrm{O}^{2-}$ ions (O13, O24, 056), which also bridge pairs of $\mathrm{Mn}(\mathrm{III})$ ions (Mn1-Mn3; Mn2-Mn4; Mn5-Mn6) to give a

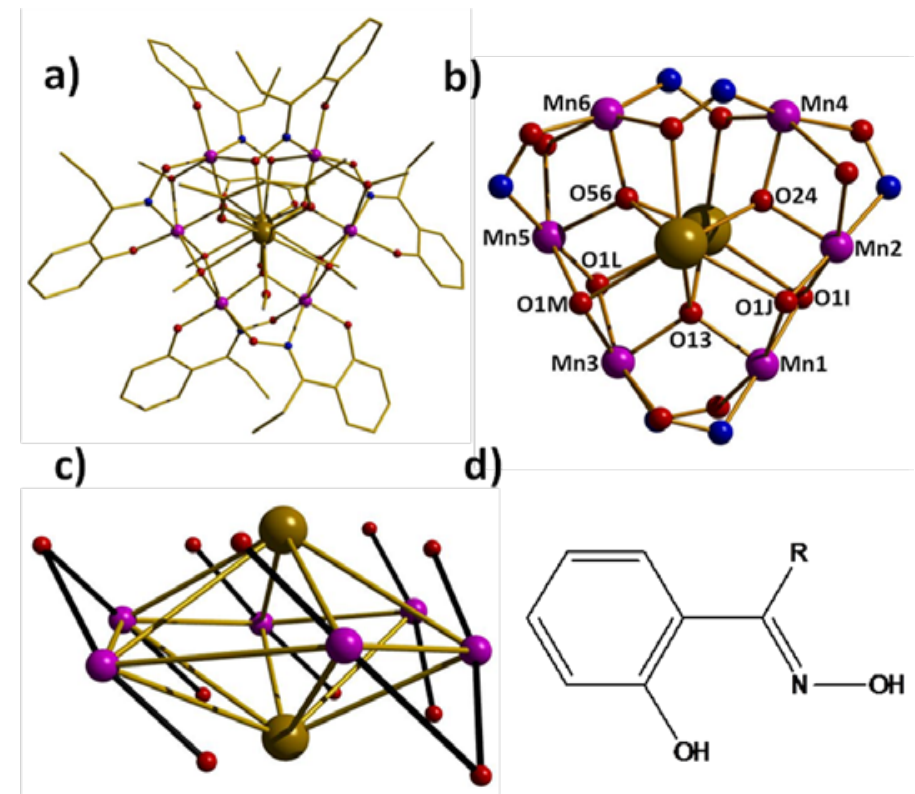

Figure 1. a) The molecular structure of $\mathbf{1}$ viewed down the Gd...Gd vector; H-atoms omitted for clarity. b) Magnetic core of $\mathbf{1}$. c) The 55 metallic skeleton of $\mathbf{1}$ viewed perpendicular to the $\mathrm{Mn}^{\mathrm{III}}{ }_{6}$ wheel with the Jahn-Teller axes of the $\mathrm{Mn}(\mathrm{III})$ ions highlighted in black. d) Generic structure of the phenolic oxime, $\mathrm{R}-\mathrm{saoH}_{2} ; \mathrm{R}=\mathrm{H}, \mathrm{saoH}_{2} ; \mathrm{R}=$ Me, $\mathrm{Me}-\mathrm{saoH}_{2} ; \mathrm{R}=\mathrm{Et}$, Et-saoH ${ }_{2}$.

$60\left[\mathrm{Gd}_{2}^{\mathrm{III}} \mathrm{Mn}_{6}^{\mathrm{III}} \mathrm{O}_{3}\right]^{18+}$ central core. The $\mathrm{Gd}$ and $\mathrm{Mn}$ ions are further linked by four $\mu_{3}-\mathrm{MeO}^{-}$ligands (O1I, O1J, O1L, O1M; Mn-O-Mn, 92.5-95.9 ; Mn-O-Gd, 97.3-101.6 ${ }^{\circ}$ ) all of which cap triangular faces on the "same side" of the trigonal prism (with respect to a plane through Mn2, Mn5, Gd1, Gd2) 65 affording the asymmetrically bridged $\left[\mathrm{Gd}^{\mathrm{III}}{ }_{2} \mathrm{Mn}^{\mathrm{III}}{ }_{6} \mathrm{O}_{3}(\mathrm{MeO})_{4}\right]^{14+}$ moiety. The Et-sao ${ }^{2-}$ ligands are of two types: four bond in the common $\eta^{1}: \eta^{1}: \eta^{1}: \mu$-fashion between neighbouring $\mathrm{Mn}(\mathrm{III})$ ions in the $\left[\mathrm{Mn}_{6}\right]$ wheel while the remaining two bond in a $\eta^{1}: \eta^{1}: \eta^{2}: \mu_{3}$-fashion between Mn4, $70 \mathrm{Mn} 6$ and Gd1; and Mn4, Mn6 and Gd2. The Mn-N-O-Mn torsion angles fall into three distinct categories: those that bridge between Mn4 and Mn6 are very large/puckered (72.5$73.3^{\circ}$ ), those that bridge between Mn2-Mn4 and Mn5-Mn6 are very small/flat $\left(4.73-7.42^{\circ}\right)$, and those between Mn1-Mn3 are 75 intermediate, but still rather large (29.5-34.5 $)$.

The Gd(III) ions are 9-coordinate and in distorted monocapped square-antiprismatic geometries with their coordination spheres each completed by the presence of one chelating acac $^{1-}$ ligand and one terminally bonded $\mathrm{MeOH}$ 80 molecule. The Mn ions are all six-coordinate and in distorted 

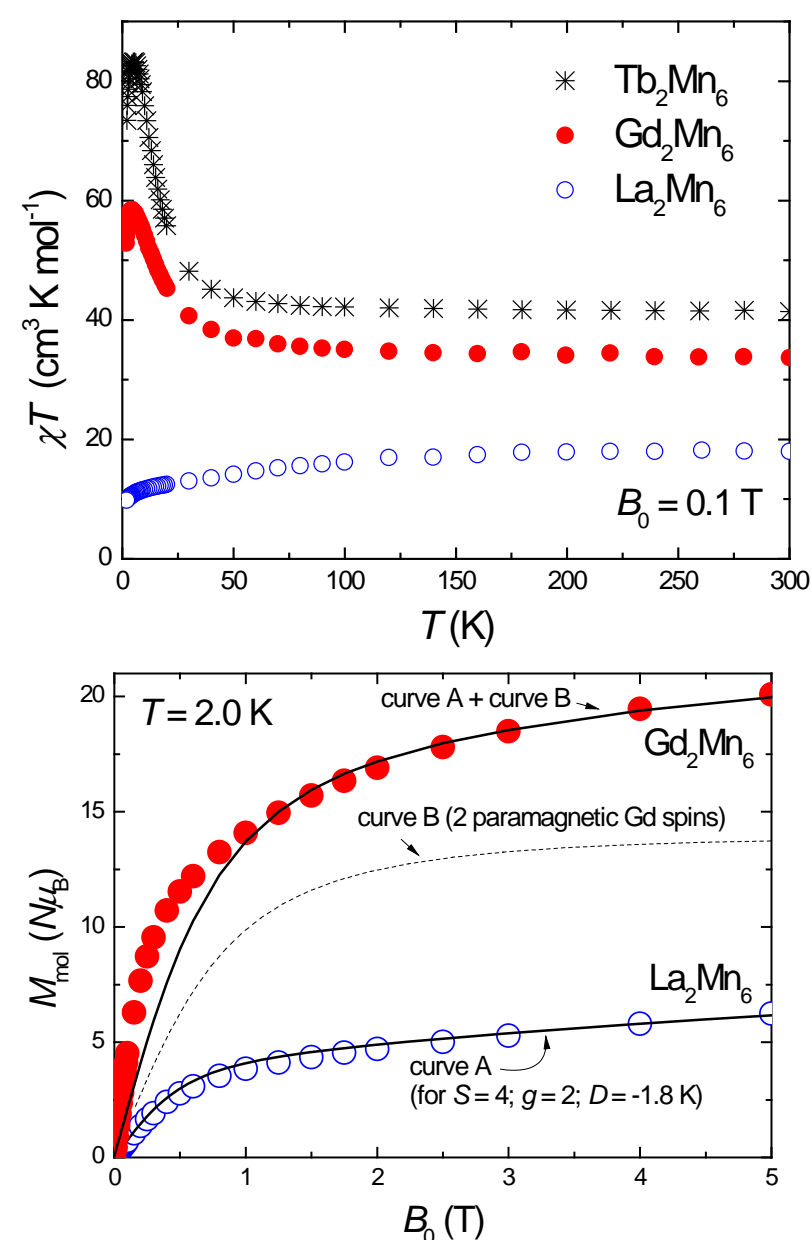

Figure 2. Plot of $\chi_{M} T$ versus $T$ for complexes 1-3 in an applied field of $0.1 \mathrm{~T}$ (top). Plot of $M_{\mathrm{mol}} / N \mu_{B}$ versus $B_{0}$ for $\mathbf{1}$ (blue) and 2 (red) at $T$ $=2 \mathrm{~K}$ (bottom). The data for 2 can be fitted to an axial zero-field splitting Hamiltonian to afford $S=4, g=2.00$ and $D=-1.8 \mathrm{~K}$ (curve $5 \mathrm{~A}$ ). The magnetisation of $\mathbf{1}$ above $1 \mathrm{~T}$ can be reproduced well by adding the calculated magnetisation of two paramagnetic Gd(III) ions (curve B) to the magnetisation data for 2.

octahedral geometries, with the exception of Mn4 and Mn6 10 which are both 5-coordinate and square-pyramidal with a longer sixth axial contact to the O-atom of a terminally bonded $\mathrm{MeOH}$ molecule (Mn4...O1K, 2.525Å; Mn6...O1N, $2.735 \AA$ ). The $\mathrm{O}$-atoms of the $\mathrm{MeOH}$ molecules are also $\mathrm{H}$ bonded to the O-atoms of the acac ${ }^{1-}$ ligands $(\mathrm{O} 1 \mathrm{~N} \ldots \mathrm{O} 4 \mathrm{H}$, $152.697 \AA)$ and terminally bonded oximic O-atoms (O1P...O23C, 2.568 $\AA$ ). All six $\mathrm{Mn}$ ions are in the III+ oxidation state, as confirmed by charge balance and bond length considerations, and BVS calculations. The Jahn-Teller axes of the six $\mathrm{Mn}(\mathrm{III})$ ions occupy the plane perpendicular 20 the $\left[\mathrm{Mn}_{6}\right]$ wheel, but are not co-parallel (Figure 1 ). In the crystal there are no significant inter-molecular H-bonds, with the closest inter-cluster contacts being of the order of $3.6 \AA$ between methoxide carbon atoms and phenolic oxygen atoms. [Mn(III)Gd(III)] clusters are surprisingly uncommon - a ${ }_{25}$ CCDC search returning only sixteeen hits. There are none with the $\left[\mathrm{Mn}(\mathrm{III})_{6} \mathrm{Gd}(\mathrm{III})_{2}\right]$ stoichiometry and none whose metallic skeleton describes a hexagonal prism. Indeed the majority possess very irrgeular topologies.
The magnetic properties of $\mathbf{1}$ were investigated by 30 dc-susceptibility measurements in the $300-2 \mathrm{~K}$ temperature range in an applied field of $0.1 \mathrm{~T}$ (Figure 2). The experimental room temperature $\chi_{M} T$ value of $\sim 33.6 \mathrm{~cm}^{3} \mathrm{~K} \mathrm{~mol}^{-1}$ is close to the spin-only $(g=2.0)$ value expected for an uncoupled [ $\mathrm{Mn}_{6}{ }_{6} \mathrm{Gd}_{2}{ }_{2}$ ] unit of $\sim 33.75 \mathrm{~cm}^{3} \mathrm{~K} \mathrm{~mol}^{-1}$. The value stays 35 essentially constant as the temperature is decreased until approximately $100 \mathrm{~K}$, below which it increases reaching a maximum value of $\sim 58.4 \mathrm{~cm}^{3} \mathrm{~K} \mathrm{~mol}^{-1}$ at $4.0 \mathrm{~K}$. Below this temperature $\chi_{M} T$ decreases reaching a value of $\sim 52.8 \mathrm{~cm}^{3} \mathrm{~K}$ $\mathrm{mol}^{-1}$ at $1.8 \mathrm{~K}$, likely a result of magnetic anisotropy and/or 40 inter-molecular anti-ferromagnetic exchange interactions. Figure 2 shows the measured molar magnetisation $M_{\text {mol }}$ in fields up to $5 \mathrm{~T}$ for $T=2 \mathrm{~K}$. The data show a rapid initial rise in magnetisation, with the high field data tending towards saturation at $\sim 20 N \mu_{B}$. Assuming that the larger Mn-O-N-Mn 45 angles favour ferromagnetic exchange, ${ }^{8}$ the dc data are thus suggestive of the two Gd(III) ions being very weakly coupled to an antiferromagnetic $(S=4)\left[\mathrm{Mn}(\mathrm{III})_{6}\right]$ wheel $(\mathrm{Mn} 1, \mathrm{Mn} 3$, Mn4, Mn6 “spin-up”, Mn2, Mn5 “spin-down”).

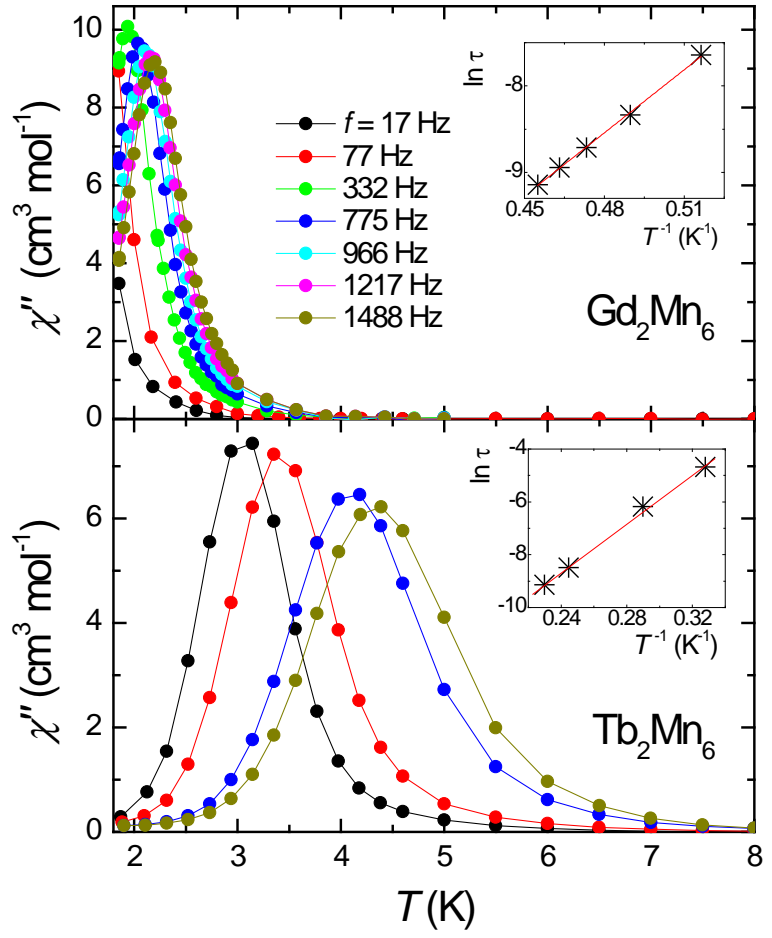

Figure 3. Plot of the out-of-phase $\left(\chi_{\mathrm{M}}{ }^{\prime \prime}\right)$ ac susceptibility for $\mathbf{1}$ (top) and $\mathbf{3}$ 50 (bottom) measured at the indicated temperatures and frequencies with an applied field of $3.5 \mathrm{G}$. The insets are a fit of the $\chi_{\mathrm{m}}$ " data to the Arrhenius Law to afford $U_{\text {eff }}=24 \mathrm{~K}$ for 1 and $U_{\text {eff }}=46 \mathrm{~K}$ for 3.

In order to address this speculation the $\mathrm{La}(\mathrm{III})\left(f^{0}\right)$ analogue of 55 complex 1, $\left[\mathrm{La}_{2} \mathrm{Mn}_{6} \mathrm{O}_{3}(\mathrm{OMe})_{4}(\text { Et-sao })_{6}(\mathrm{acac})_{2}(\mathrm{MeOH})_{4}\right] \cdot \mathrm{C}_{6} \mathrm{H}_{14}$ (2.hex) was synthesised. The structure ${ }^{\dagger \ddagger}$ of complex 2 is analogous to $\mathbf{1}$, with small changes to the Mn-N-O-Mn torsion angles. The experimental room temperature $\chi_{M} T$ value of $\sim 18 \mathrm{~cm}^{3}$ $\mathrm{K} \mathrm{mol}^{-1}$ for 2 is as expected for an uncoupled [ $\mathrm{Mn}_{6}^{\mathrm{III}}$ ] unit with $g$ ${ }_{60}=2.00$. The value drops steadily with temperature to a value of approximately $10 \mathrm{~cm}^{3} \mathrm{~K} \mathrm{~mol}^{-1}$ at $2 \mathrm{~K}$. The low temperature value is suggestive of $S=4$. The magnetisation data for 2 at $2 \mathrm{~K}$ is 
shown in the lower panel of Figure 2 and can be fitted to an axial zero-field splitting plus Zeeman Hamiltonian $\left(H=D S_{z}^{2}+\right.$ $g \mu_{\mathrm{B}} B_{0} S$ ) to yield $S=4, g=2.00$ and $D=-1.8 \mathrm{~K}$. The magnetisation data for $\mathbf{1}$ can be well reproduced above $B_{0} \sim 1 \mathrm{~T}$ 5 by adding two Brillouin functions (“curve B” in Figure 2) for two paramagnetic Gd(III) ions to the magnetisation data for 2 . Figure 2 shows that fields lower than $1 \mathrm{~T}$ do not decouple the Gd(III) ions from the inner $\left[\mathrm{Mn}(\mathrm{III})_{6}\right]$ wheel, and that this coupling is ferromagnetic since the experimental $M_{\text {mol }}$ for $\mathbf{1}$ lies above the 10 calculated curve in this field region.

The relatively large and anisotropic spin associated with complexes 1 and $\mathbf{2}$ suggest that they are likely to be SMMs, and this is confirmed through the appearance of frequency-dependent out-of-phase $\left(\chi_{\mathrm{M}}{ }^{\prime \prime}\right)$ signals for both. For 2 only the tails of the 15 peaks are observed, but fully visible peaks are seen for $\mathbf{1}$ in the 332-1488 Hz range between 2 and $4 \mathrm{~K}$ (Figure 3). The difference between the two complexes is simply attributed to the differences in the Mn-N-O-Mn torsion angles which are smaller in magnitude for $\mathbf{2}$ than for $1 .^{8}$ The $\chi_{\mathrm{M}}{ }^{\prime \prime}$ data obtained for $\mathbf{1}$ were fit to the 20 Arrhenius equation to give an effective energy barrier, $U_{\text {eff }}=24 \mathrm{~K}$ with $\tau_{0}=1.7 \times 10^{-9} \mathrm{~s}$. In order to enhance the energy barrier to magnetisation relaxation the $\mathrm{Tb}(\mathrm{III})$ analogue of complex $\mathbf{1}$, $\left[\mathrm{Tb}_{2} \mathrm{Mn}_{6} \mathrm{O}_{3}(\mathrm{OMe})_{4}(\mathrm{Me}-\mathrm{sao})_{6}(\mathrm{acac})_{2}(\mathrm{MeOH})_{4}\right] \cdot \mathrm{C}_{6} \mathrm{H}_{14}$ (3·hex) was synthesised. The structure ${ }^{\dagger \ddagger}$ of complex $\mathbf{3}$ is again analogous to $\mathbf{1}$, 25 but with $\mathrm{Me}-\mathrm{sao}^{2-}$ replacing Et-sao ${ }^{2-}$ and with minor changes to bond lengths and angles. ${ }^{\ddagger}$ As illustrated in Figure 3, the effect on the relaxation behaviour is rather dramatic. Fully visible out-ofphase $\chi_{\mathrm{M}}$ " signals are now seen for all the frequencies employed (including $17 \mathrm{~Hz}$ ) and each signal is shifted to higher 30 temperatures; indeed the $1488 \mathrm{~Hz}$ signal is shifted by approximately $2.5 \mathrm{~K}$. The result is that the energy barrier to magnetisation reversal in complex $3\left(U_{\text {eff }}=46.3 \mathrm{~K}\right)$ is effectively double that of complex 1.

In conclusion, we have reported the first examples of $3 d-4 f$ 35 clusters built with derivatised saliclyaldoximes - unusual $\left[\mathrm{Ln}_{2}^{\mathrm{III}} \mathrm{Mn}_{6}{ }_{6}\right.$ ] hexagonal prisms. Replacement of the paramagnetic $\mathrm{Gd}(\mathrm{III})$ ions with the diamagnetic La(III) ions confirms the presence of an antiferromagnetic $\left[\mathrm{Mn}_{6}^{\mathrm{III}}{ }_{6}\right.$ ] wheel coupled very weakly to two apical Gd(III) ion caps. The anisotropic spin 40 renders 1 and 2 SMMs and replacement of Gd(III) with Tb(III) in

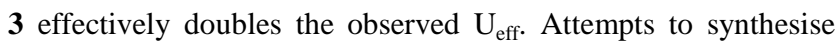
and characterise all the post-Gd versions of $\mathbf{1}$ are in progress.

\section{Notes and references}

${ }^{a}$ Université de Reims Champagne-Ardenne, Institut de Chimie ${ }_{45}$ Moléculaire de Reims (ICMR), Groupe Chimie de Coordination, CNRS UMR 6229, Bât. Europol'Agro - Moulin de la Housse, BP 1039 - 51687 Reims cedex 2, France

${ }^{b}$ EaStCHEM School of Chemistry, The University of Edinburgh, West Mains Road, Edinburgh, EH9 3JJ (UK). Fax: (+44)131-650-6453; E50 mail: ebrechin@staffmail.ed.ac.uk

${ }^{c}$ Instituto de Ciencia de Materiales de Aragón, CSIC-Universidad de Zaragoza, Departamento de Física de la Materia Condensada, 50009 Zaragoza, Spain,E-mail: evange@unizar.es

$55 \dagger$ The complex analysed as $10.7 \mathrm{MeCN}$. Elemental analysis, expected (found): C, 40.82 (41.11); H, 4.41 (4.60); N, 4.57 (4.76)\%. Diffraction data were collected with $\mathrm{Cu}$ radiation $(\lambda=1.5418 \AA)$ on an Oxford
Diffraction SuperNova Dual wavelength diffractometer with an Atlas CCD detector. Crystal data: $\mathrm{C}_{73.4} \mathrm{H}_{94.1} \mathrm{Gd}_{2} \mathrm{Mn}_{6} \mathrm{~N}_{6.7} \mathrm{O}_{27}, M=2146.39$, red 60 block, monoclinic, P 21/c, $a=26.3789(3), b=14.04527(13), c=$ 25.8211(3) $\AA, \alpha=90.0, \beta=117.7884(14), \gamma=90.0^{\circ}, V=8463.41(15) \AA^{3}$, $Z=4, T=100 \mathrm{~K}, 83524$ reflections collected of which 16710 were independent $\left(R_{\mathrm{int}}=0.0937\right), 1049$ parameters and 4 restraints, $R 1=$ 0.0445 [based on $F>4 \sigma(F)$ ], $w R 2=0.1151$ (based on $F^{2}$ and all data). 65 CCDC XXXXXX.

The complex analysed as $2 \mathrm{C}_{6} \mathrm{H}_{14}$. Elemental analysis, expected (found): C, 43.25 (43.40); H, 4.89 (4.85); N, 3.88 (3.84)\%. Diffraction data were collected with Mo-K $\alpha$ radiation $(\lambda=0.71073 \AA$ ) on a Bruker Smart Apex CCD diffractometer. Crystal data: $\mathrm{C}_{78} \mathrm{H}_{105} \mathrm{La}_{2} \mathrm{Mn}_{6} \mathrm{~N}_{6} \mathrm{O}_{27}, M=2166.15$, 70 black cuboid, orthorhombic, $C 2222_{1}, a=15.3154(6), b=22.8884(6), c=$ 25.7786(8) $\AA, \alpha=\beta=\gamma=90.0^{\circ}, V=9036.6(5) \AA^{3}, Z=4, T=100 \mathrm{~K}$, 66786 reflections collected of which 9309 were independent $\left(R_{\text {int }}=\right.$ 0.0391), 766 parameters and 505 restraints, $R 1=0.0223$ [based on $F>$ $4 \sigma(F)], w R 2=0.0578$ (based on $F^{2}$ and all data). CCDC XXXXXX.

1. (a) A. Bencini, C. Benelli, A. Caneschi, R. L. Carlin, A. Dei and D. Gatteschi, J. Am. Chem. Soc., 1985, 107, 8128; (b) Y. Pei, Y. Journaux, O. Kahn, A. Dei and D. Gatteschi, J. Chem. Soc., Chem. Commun., 1986, 1300; (c) C. Benelli, A. Caneschi, D. Gatteschi, O.

80 Guillou and L. Pardi, Inorg. Chem., 1990, 29, 1750; (d) R. E. P. Winpenny, Chem. Soc. Rev., 1998, 27, 447.

2. (a) R. Sessoli, D. Gatteschi and J. Villain, Molecular Nanomagnets, Oxford University Press, 2006; (b) G. Aromí and E. K. Brechin, Struct. Bonding, 2006, 122, 1; (c) M. Murrie and D. J. Price, Annu. Rep. Prog. Chem., Sect. A, 2007, 103, 20; (d) A. K. Boudalis, Y. Sanakis, C. P. Raptopoulou, V. Psycharis, Magnetism and Superconductivity in Low-Dimensional Systems: Utilization in Future Applications, D. Stamopoulos (Ed.) Nova Science Publishers Inc., New York, 2008, pp. 1-77; (e) G. Christou, Polyhedron, 2005, 24, 2065.

3. M. Evangelisti, F. Luis, L. J. de Jongh and M. Affronte, J. Mater. Chem., 2006, 2534.

4. R. Sessoli and A. K. Powell, Coord. Chem. Rev., 2009, 253, 2328 and references therein.

95 5. (a) N. Ishikawa, M. Sugita, T. Ishikawa, S. Koshihara and Y. Kaizu, J. Am. Chem. Soc., 2003, 125, 8694; (b) M. A. AlDamen, J. M. Clemente-Juan, E. Coronado, C Martí-Gastaldo and A. Gaita-Ariño J. Am. Chem. Soc., 2008, 130, 8874.

6. M. Evangelisti and E. K. Brechin, Dalton Trans., 2010, 20, 4672 and references therein.

7. (a) G. Karotsis, S. Kennedy, S. J. Teat, C. M. Beavers, D. A. Fowler, J. J. Morales, M. Evangelisti, S. J. Dalgarno and E. K. Brechin, J. Am. Chem. Soc., 2010, 132, 12983; (b) Y.-Z. Zheng, M. Evangelisti and R. E. P. Winpenny, Chem. Sci., 2011, 99.

105 8. (a) R. Inglis, S. M. Taylor, L. F. Jones, G. S. Papaefstathiou, S. P. Perlepes, S. Datta, S. Hill, W. Wernsdorfer and E. K. Brechin, Dalton Trans., 2009, 9157; (b) R. Inglis, L. F. Jones, C. J. Milios, S. Datta, A. Collins, S. Parsons, W. Wernsdorfer, S. Hill, S. P. Perlepes, S. Piligkos and E. K. Brechin, Dalton Trans., 2009, 3403; (c) C. J. Milios, S. Piligkos and E. K. Brechin, Dalton Trans., 2008, 1809; (d) C. J. Milios, R. Inglis, A. Vinslava, R. Bagai, W. Wernsdorfer, S. Parsons, S. P. Perlepes, G. Christou and E. K. Brechin, J. Am. Chem. Soc., 2007, 129, 12505; (e) C. J. Milios, A. Vinslava, W. Wernsdorfer, A. Prescimone, P. A. Wood, S. Parsons, S. P. Perlepes, G. Christou and E. K. Brechin, J. Am. Chem. Soc., 2007, 129, 6547.

9. M. Andruh, J. P. Costes, C. Diaz and S. Gao, Inorg. Chem., 2009, 48, 3342 and references therein. 